\title{
Breast artery calcification as a predictor of coronary artery calcification: a cross-sectional study
}

\author{
Batool Seifi ${ }^{1 A, C, F}$, Reza Javadrashid ${ }^{2 A, B}$, Fatemeh Seifi ${ }^{1 C, D, F}$, Jhila Khamanian ${ }^{3 B, F}$, Armin Zarrintan ${ }^{4 A, B, C}$, \\ Mohammad Mirza-Aghazadeh-Attari ${ }^{4,5 A, C, D, E, F}$ \\ 'Department of Radiology, Faculty of Medicine, Tabriz University of Medical Sciences, Tabriz, Iran \\ ${ }^{2}$ Department of Radiology, Medical Radiation Sciences Research Group, Imam Reza Hospital, Tabriz University of Medical Sciences, Tabriz, Iran \\ ${ }^{3}$ Department of community and preventive medicine, Faculty of Medicine, Tabriz University of Medical Sciences, Tabriz, Iran \\ ${ }^{4}$ Medical Radiation Sciences Research Group, Department of Radiology, Imam Reza Hospital, Tabriz University of Medical Sciences, Tabriz, Iran \\ ${ }^{5}$ Aging Research Institute, Tabriz University of Medical Sciences, Tabriz, Iran
}

\section{Abstract}

Introduction: Coronary artery disease is the main cause of burden of disease in the world. Coronary calcification is seen as an aetiopathological event in the pathogenesis of cardiovascular diseases. Studies have shown that breast artery calcification, which is routinely found in mammography of elderly women, could be predictive of coronary artery calcification.

Material and methods: In this cross-sectional study, 60 women over 40 years of age were included. All of these patients had undergone mammography after having an indication to undergo a computed tomography-angiography. Breast arterial calcification and calcium scores were determined for each patient, and the paired- $t$ test was used to analyse the data.

Results: The mean age of patients was $49.52 \pm 8.83$ years. Of these 60 women, $50 \%$ were postmenopausal and $50 \%$ were not. In $37(61.7 \%)$ cases, mild to severe coronary calcification was observed, and $50(83.3 \%)$ had mild to severe breast arterial calcification. There was a significant correlation between coronary calcification and breast artery calcification ( $p=0.001$ ), and there was also a significant relationship between coronary calcification and postmenopausal calcification $(p<0.001)$.

Conclusions: Breast artery calcification can be a suitable predictor for coronary artery calcification and is a valid method for predicting cardiovascular disease probability in the future.

Key words: calcification, coronary artery, CT angiography, mammogram, breast.

\section{Introduction}

Mammography is the routine method for breast cancer screening. This modality is able to elicit calcifications inside breast tissue. These calcifications can be seen in two general forms including micro-calcification and macrocalcification. Macro-calcification is usually associated with benign lesions, while micro-calcification is associated with neoplastic lesions [1]. Another imaging finding seen in mammography is the calcification of breast arteries (or breast arterial calcification - BAC). This finding is regarded as a normal phenomenon in elderly post-menopausal women [2]. BACs are radio-opaque linear lesions that are not situated near the areola [3]. Descriptive studies have shown that BACs are commonly seen in elderly women with stablished risk factors for arteriosclerosis. Unlike typical arteriosclerotic lesions, calcium is deposited in the media of the arteries, instead of the endothelium. Early

\section{Correspondence address:}

Dr. Mohammad Mirza-Aghazadeh-Attari, Aging Research Institute, Tabriz University of Medical Sciences, Tabriz, Iran, e-mail: m.aghazadeh75@yahoo.com Authors' contribution:

A Study design · B Data collection · C Statistical analysis · D Data interpretation · E Manuscript preparation · F Literature search · G Funds collection 
observational studies have been able to detect a link between the existence of BAC and calcification of coronary arteries, which is diagnosed by computed tomography (CT)-angiography and is shown to be significantly associated with increased cardiovascular diseases [4], although these observations have been challenged by case-control and cross-sectional studies in which there has been no significant relation between BAC and coronary calcification. BAC in particular has also been directly linked with an increased risk of acute myocardial infarction, although observations have not yielded consistent results [5]. Because of the regularity with which mammography is performed, especially in developed nations, and the possible predictable role of BAC in cardiovascular disease, some have suggested that mammography could be used as a surrogate screening method for cardiovascular diseases. But this desire is not limited to academic scholars, as a study performed by Margolies et al. suggested that patients would also prefer to be notified about possible cardiovascular diseases using BAC estimation [6]. In the present study we aim to assess the relation between BAC and coronary calcium score, and also the association of BAC with risk factors of cardiovascular diseases.

\section{Material and methods}

\section{Patients}

The present cross-sectional study was performed in a tertiary medical centre, which was a referral centre for cardiovascular diseases. This study included 60 patients who were referred to undergo CT-angiography. Inclusion criteria consisted of being referred to our centre with a diagnosis of coronary artery disease by a cardiologist. Exclusion criteria consisted of having a past medical history of breast cancer and not wanting to participate in the study.

\section{Sample size calculation}

Sample size was calculated by the following formula:

Power $=80 \%, \alpha=0.5 \rightarrow Z \alpha=1.96, \beta=0.8 \rightarrow Z \beta=0.842$, $\mathrm{C}=0.5^{\star} \ln \left[(1+\mathrm{r}) /(1-\mathrm{r}), \mathrm{N}=[(Z \alpha+Z \beta) / \mathrm{C}]^{2}+3=53\right.$. In this study, 60 patients were included.

Table 1. Time interval between computed tomography (CT)-angiography and mammography in patients

\begin{tabular}{|l|c|}
$\begin{array}{l}\text { Time interval between CT-angiography } \\
\text { and mammography (months) }\end{array}$ & $n(\%)$ \\
\hline 2 & $7(11.7)$ \\
\hline 3 & $8(13.3)$ \\
\hline 4 & $20(33.3)$ \\
\hline 5 & $16(26.7)$ \\
\hline 6 & $9(15)$ \\
\hline
\end{tabular}

\section{Calcium scoring}

Calcium scoring was performed according to the latest guideline published by the cardiac society of Australia and New Zealand [7]. Calcium score was calculated by determining the area of calcification and multiplying it to the highest Hounsfield unit. Calcium score was classified into five sets of values, equalling zero, between 1 and 10, between 11 and 100, between 101 and 400, and more than 400. To determine this score, a 64-slice CT-angiography machine (Siemens, Germany) was used.

\section{Breast artery calcification evaluation}

$\mathrm{BAC}$ is evaluated by determining the density, number, and length of calcified vessels. Each of these parameters are attributed a score, and the sum of these determines the BAC value. BAC values are categorised into three subgroups, BAC equalling zero, between 1 and 6 , and between 7 and 12. In this study, we used a simpler method for BAC classification. Mild BAC was defined as scant calcification in vessels, in which the distance between the calcifications was at least $10 \mathrm{~cm}$. Moderate $\mathrm{BAC}$ was defined as the calcification of the complete length of an artery, and sever $\mathrm{BAC}$ was defined as having complete calcification in multiple arteries.

Mammographies were obtained using the Hologic Dimension unit (Bedford, MA, USA).

\section{Statistical analysis}

IBM SPSS version 20 (IBM, Armonk, NY) was used to analyse the data. The power of the study was set at $80 \%$ and $p$ less than 0.05 was considered significant. Paired $t$-test was used to analyse the relation between BAC values and calcium scores.

\section{Results}

The mean age of patients included in the study was 49.52 \pm 8.83 years. From all of the patients, 30 had experienced menopause. Only one patient had a history of cigarette smoking. The time period between the CT angiography and the mammography is documented in Table 1. As it can be seen the most common time interval was four months.

Table 2 depicts the calcium scores and BAC values documented for the patients. Of all the patients, 37 had evidence of calcification in their coronary arteries and 50 patients had evidence of breast artery calcification. Paired $t$-test showed that there was a significant relation between having coronary calcification and breast artery calcification $(p=0.001)$.

There was no significant relation between menopausal women and those with ongoing ovulatory cycles regarding the existence of calcified coronary or breast arteries. There was a significant relation between the existence of risk fac- 
tors such as diabetes, hypertension, and hyperlipidaemia and calcified breast and coronary arteries $(p<0.0001)$.

The relation between the severity of calcification of the breast arteries and the coronary arteries was also determined. There was a significant relation between the increase in one value and the increase in the other $(p<0.001)$.

Figure 1 is a schematic presentation of breast artery calcification staging. Figure 2 demonstrates selected coronary calcification depictions of the patients included in the study. Figure 3 shows breast artery calcification in selected patients who were included in the study.
Table 2. Calcium scores and breast artery scores of patients included in the study

\begin{tabular}{|c|c|c|c|c|c|}
\hline \multirow{2}{*}{$\begin{array}{l}\text { Calcium } \\
\text { score }\end{array}$} & \multicolumn{4}{|c|}{ BAC grades } & \multirow[t]{2}{*}{ Total } \\
\hline & None & Mild & Moderate & Severe & \\
\hline 0 & 7 & 16 & 0 & 0 & 23 \\
\hline 1 & 2 & 13 & 0 & 0 & 15 \\
\hline 2 & 1 & 9 & 4 & 0 & 14 \\
\hline 3 & 0 & 1 & 4 & 1 & 6 \\
\hline 4 & 0 & 0 & 1 & 1 & 2 \\
\hline Total & 10 & 39 & 9 & 2 & 60 \\
\hline
\end{tabular}

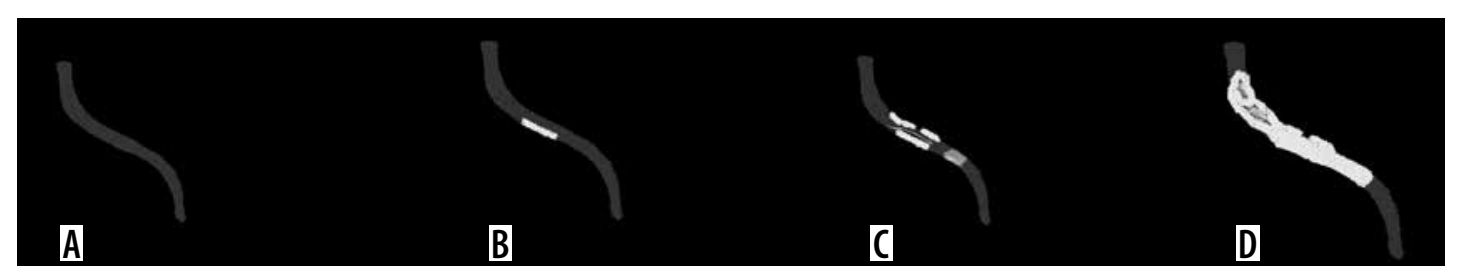

Figure 1. Schematic presentation of breast arterial calcification. The figures indicate increasing grade from $\mathbf{A}$ to $\mathbf{D}$. The grading is done based on severity and density of calcium deposition in breast arteries
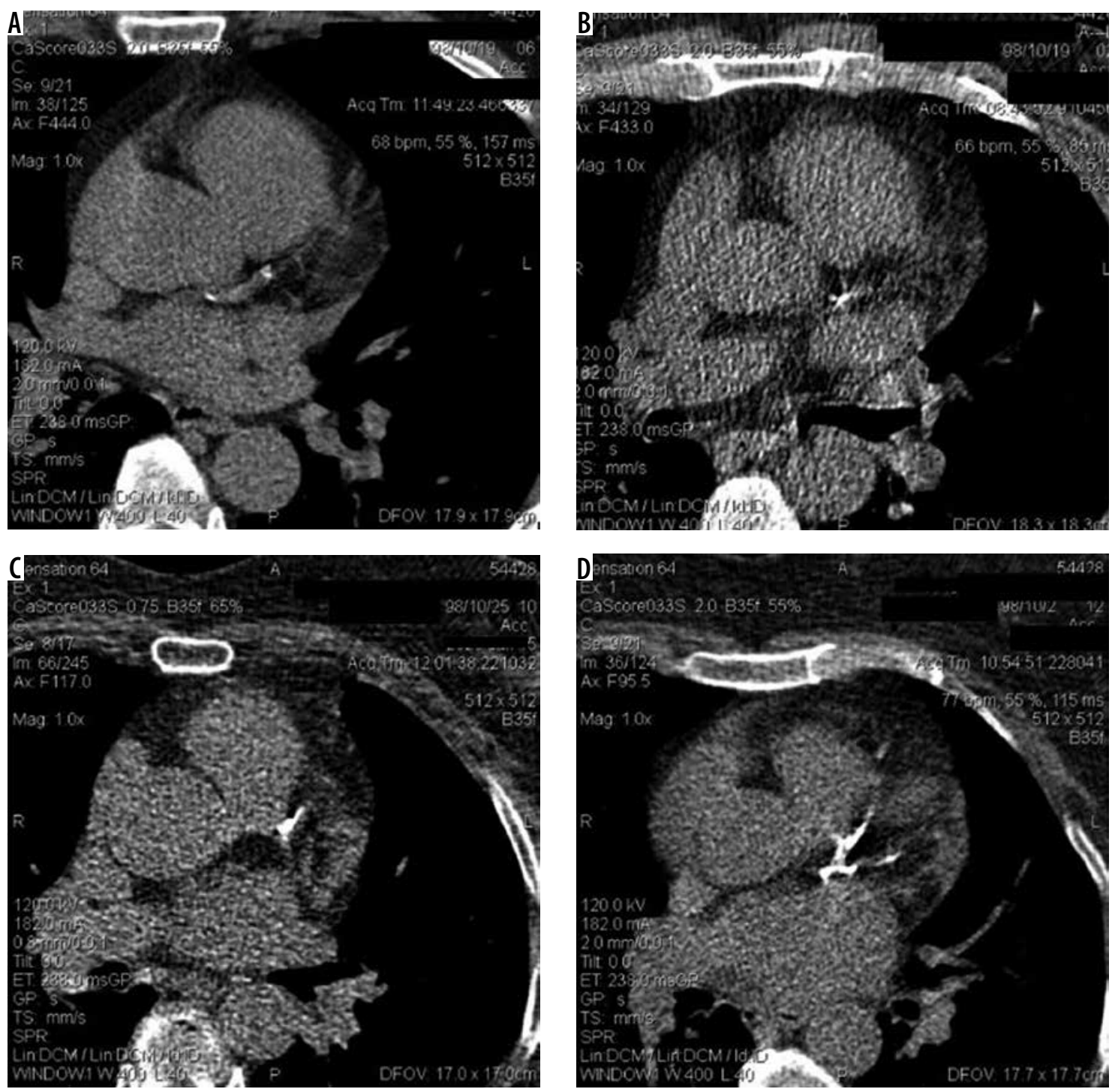

Figure 2. Coronary calcification score depicted in four female patients. A) Patient with calcium score of 1-10. B) Patient with calcium score of 11-100. C) Patient with calcium score of 101-400. D) Patient with calcium score of more than 400 

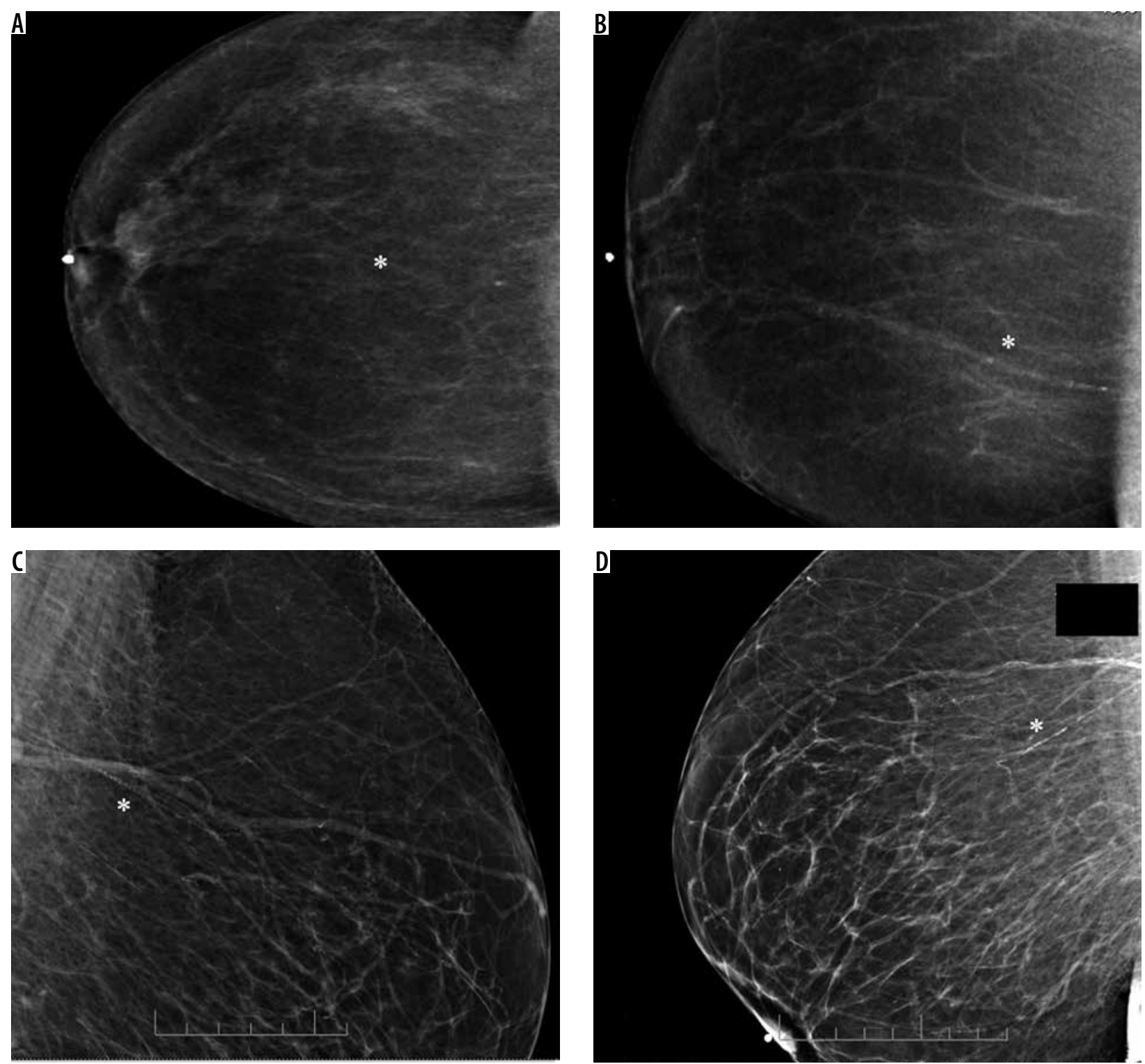

Figure 3. Breast artery calcification. A) Grade 1. B) Grade 2. C) Grade 3. D) Grade 4. Asterisks shows the point of interest in all four pictures

\section{Discussion}

There has long been a discussion regarding the clinical significance of calcification of the media of the arteries, and its compression to intimal calcification. Although it has been known that medial thickening of the artery is associated with high blood pressure, not much is known about how medial thickness contributes to coronary heart disease, or whether it is related with intimal calcification, which is proven to be associated with cardiovascular disease [8]. Importantly, observational studies have reported that similar risk factors exist for intimal and medial thickness and calcification [9]. Because of these links between the two types of calcification, it is hypothesised that vascular calcifications of both kinds could be related and be of prognostic and diagnostic value towards each other.

Mammography is currently the main method of breast cancer screening in women over 40 years old, and guidelines suggest annual imaging after a certain age, with the exact age cut-off still a controversy [10]. Mammography is able to show breast artery calcification of medial type, and could be associated with intimal thickness in coronary arteries, which is shown to be an aetiopathological starting point for the progression of coronary artery disease, the main cause of global burden of disease [11].

An early study performed by Henkin et al. studied the relation between BAC and coronary artery disease proven by angiography on 319 patients aged between 50 and 70 years but did not find an association. The patients with coronary artery disease had an increased prevalence of risk factors for ischaemic heart disease, and a marginally increased rate of BAC, but the difference between the two groups was not significant. They found that the existence of hypertension and being aged above 63 years were significantly related to the existence of BAC [12]. This finding could be of significance, knowing that most breast cancer screening programs continue until the age of 63 years, but most not much further [13]. Another study performed by 
Pecchi et al. found that BAC was associated with coronary artery disease in a group of post-menopausal women, a group of patients with a higher mean age [14]. Penugonda et al. suggested that BAC did not even correlate with risk factors associated with coronary artery disease, and that it was not significantly related with symptomatic coronary disease. They even suggested that patients with BAC had lower prevalence of myocardial infarction, compared to those without BAC [15]. Moradi et al. conducted a similar study and found similar results [16]. Matsumura et al. found the exact opposite in asymptomatic women - they found that patients with BAC had an odds ratio of over 22 to have calcium scores more than 400 . This association was not witnessed in patients with calcium scores of 0 , suggesting that the absence of BAC is not as suggestive of a coronary condition as its existence [17]. Again, this negative predicting role may be of significance in elder populations, though no study has focused on this issue so far.

Another issue which is suggested to play a role in the relation between $\mathrm{CAD}$ and $\mathrm{BAC}$ is race; all of the previously mentioned articles were performed on Caucasian populations. Newallo et al. studied a group consisting of African American women with a median age of 52.5 years. In the 204 patients included, 42 had BAC in mammography. There was a significant relation between the existence of BAC and calcium scores of more than 100, luminal narrowing, and stenosis of over $50 \%$ of coronary vessels [18].

Calcium scores of over 400 are usually found to be clinically significant, but low levels of calcium score, even as low as 10 , are found to be associated with increased risk of cardiovascular accidents. A study by Chadashvili et al. showed that BAC correlated with calcium scores of more than 11. BAC in particular was related with cardiovascular risk factors, such as diabetes and renal disease [19]. Fanthala et al. reported similar results in a retrospective study performed on 307 Arab women [20].

A recent large-scale cohort study performed on 5145 people aged between 60-79 years and of different ethnicities was performed to assess the relation between BAC and cardiovascular diseases and risk factors. It was found that as age increased, the probability of finding BAC also increased (19\% in women aged between 60 and 64 years compared to $52 \%$ in those aged $75-79$ years). The intensity of BAC also increased with age. BAC was more common in Hispanic populations, followed by Caucasians, Blacks, and
Asians. The first publication from this study focuses on the recruitment characteristics, and future publications will guide clinicians and researchers in better understanding the predictive role of BAC in cardiovascular diseases [21].

A recent review on the possible applications of BAC in cardiovascular disease risk stratification proposed that, although the concept of using BAC for CVD was appealing, there were major barriers to overcome. One barrier was the technical heterogeneity in which the measurements are made, and also the different cut-off values defined for increased calcium score values (as seen before, different scholars have used values ranging from 10 to more than 400 to define a clinically significant positive calcium score). Another noteworthy limitation is how the progress of imaging techniques has affected BAC detection. Interestingly, newer studies tend to better detect BAC and Coronary artery disease, making old evidence less relevant, and dictating the need for more studies performed with the latest appliances available [22]. One novel aspect of technology recently applied in this regard is deep learning algorithms, which have been able to detect BACs as well as expert radiologists, and may be a significant contribution to systemic screening of women for both breast anomalies and cardiovascular diseases [23].

Altogether, compelling evidence exists to support the use of BAC in clinical practice, but there is not enough evidence to support how and to what extent BAC should be used as a risk factor for coronary artery disease, and most importantly ischaemic heart disease. Larger scale cohort studies will be of merit in this regard. Further technical notes will also be needed to formulate a standardised method for imaging of BAC and applying it in clinical contexts.

\section{Conclusions}

BAC was significantly related to the existence of coronary artery calcification detected in CT-angiography. This relation is not affected by age. BAC was also associated with an increased prevalence of coronary artery disease risk factors, such as diabetes, hyperlipidaemia, and hypertension.

\section{Conflict of interest}

The authors report no conflict of interest.

\section{References}

1. Nalawade YV. Evaluation of breast calcifications. Indian J Radiol Imaging 2009; 19: 282-286.

2. Margolis J, Chen J, Kong Y, et al. The diagnostic and prognostic significance of coronary artery calcification. A report of 800 cases. Radiology 1980; 137: 609-666.

3. Lai KC, Slanetz PJ, Eisenberg RL. Linear breast calcifications. Am J Roentgenol 2012; 199: W151-W157.
4. Neves PO, Andrade J, Monção H. Coronary artery calcium score: current status. Radiol Bras 2017; 50: 182-189.

5. Bui QM, Daniels LB. A Review of the role of breast arterial calcification for cardiovascular risk stratification in women. Circulation 2019; 139: 1094-1101.

6. Margolies LR, Yip R, Hwang E, et al. Breast arterial calcification in the mammogram report: the patient perspective. Am J Roentgenol 2019; 212: 209-214. 
7. Hamilton-Craig C, Liew G, Chan J, et al. Coronary artery calcium scoring - position statement. 2017.

8. Amann K. Media calcification and intima calcification are distinct entities in chronic kidney disease. Clin J Am Soc Nephrol 2008; 3 : 1599-1605.

9. Chen NX, Moe SM. Vascular calcification: pathophysiology and risk factors. Curr Hypertens Rep 2012; 14: 228-237.

10. Fiorica JV. Breast cancer screening, mammography, and other modalities. Clin Obstet Gynecol 2016; 59: 688-709.

11. Schenker MP, Dorbala S, Hong EC, et al. Interrelation of coronary calcification, myocardial ischemia, and outcomes in patients with intermediate likelihood of coronary artery disease: a combined positron emission tomography/computed tomography study. Circulation 2008; 117: 1693-1700.

12. Henkin Y, Abu-Ful A, Shai I, Crystal P. Lack of association between breast artery calcification seen on mammography and coronary artery disease on angiography. J Med Screening 2003; 10: 139-142.

13. Oeffinger KC, Fontham ET, Etzioni R, et al. Breast cancer screening for women at average risk: 2015 guideline update from the American Cancer Society. JAMA 2015; 314: 1599-1614.

14. Pecchi A, Rossi R, Coppi F, Ligabue G, Modena M, Romagnoli R. Association of breast arterial calcifications detected by mammography and coronary artery calcifications quantified by multislice CT in a population of post-menopausal women. La Radiologia Medica 2003; 106: 305-312.

15. Penugonda N, Billecke SS, Yerkey MW, Rebner M, Marcovitz PA. Usefulness of breast arterial calcium detected on mammography for predicting coronary artery disease or cardiovascular events in women with angina pectoris and/or positive stress tests. Am J Cardiol 2010; 105: 359-361.
16. Moradi M, Adibi A, Abedi M. Relationship between breast arterial calcification on mammography with CT Calcium scoring and coronary CT angiography results. Adv Biomed Res 2014; 3: 79.

17. Matsumura ME, Maksimik C, Martinez MW, et al. Breast artery calcium noted on screening mammography is predictive of high risk coronary calcium in asymptomatic women: a case control study. Vasa 2013; 42: 429-433.

18. Newallo D, Meinel FG, Schoepf UJ, et al. Mammographic detection of breast arterial calcification as an independent predictor of coronary atherosclerotic disease in a single ethnic cohort of African American women. Atherosclerosis 2015; 242: 218-221.

19. Chadashvili T, Litmanovich D, Hall F, Slanetz PJ. Do breast arterial calcifications on mammography predict elevated risk of coronary artery disease? Eur J Radiol 2016; 85: 1121-1124.

20. Fathala AL, Alabdulkarim FM, Shoukri M, Alanazi M. Association between breast arterial calcifications found on mammography and coronary artery calcifications in asymptomatic Saudi women. Ann Saudi Med 2018; 38: 433-438.

21. Iribarren C, Sanchez G, Husson G, et al. MultIethNic Study of BrEast ARterial calcium gradation and CardioVAscular Disease: cohort recruitment and baseline characteristics. Ann Epidemiol 2018; 28: 41-7.e12.

22. Quispe R, Al-Rifai M, Di Carlo PA, et al. Breast arterial calcium: a game changer in women's cardiovascular health? JACC Cardiovasc Imaging 2019; 12: 2538-2548.

23. Wang J, Ding H, Bidgoli FA, et al. Detecting cardiovascular disease from mammograms with deep learning. IEEE Trans Med Imaging 2017; 36: 1172-1181. 